\title{
Uniformly connected conductive networks on cellulose nanofiber paper for transparent paper electronics
}

\author{
Hirotaka Koga, Masaya Nogi, Natsuki Komoda, Thi Thi Nge, Tohru Sugahara and Katsuaki Suganuma \\ We demonstrate the fabrication of highly transparent conductive networks on a cellulose nanofiber paper, called cellulose \\ nanopaper. Uniform coating of the conductive nanomaterials, such as silver nanowires (AgNWs) and carbon nanotubes, is \\ achieved by simple filtration of their aqueous dispersions through the cellulose nanopaper, which acts as both filter and \\ transparent flexible substrate. The as-prepared AgNW networks on the nanopaper offer sheet resistance of $12 \Omega \mathrm{sq}^{-1}$ with \\ optical transparency of $88 \%$, which is up to 75 times lower than the sheet resistance on a polyethylene terephthalate film \\ prepared by conventional coating processes. These results indicate that the 'filtration coating' provides uniformly connected \\ conductive networks because of drainage in the perpendicular direction through paper-specific nanopores, whereas conventional \\ coating processes inevitably cause self-aggregation and uneven distribution of the conductive nanomaterials because of the \\ hard-to-control drying process, as indicated by the well-known coffee-ring effect. Furthermore, the conductive networks are \\ embedded in the surface layer of the nanopaper, showing strong adhesion to the nanopaper substrate and providing foldability \\ with negligible changes in electrical conductivity. This filtration process is thus expected to offer an effective coating approach \\ for various conductive materials, and the resulting transparent conductive nanopaper is a promising material for future paper \\ electronics.
}

NPG Asia Materials (2014) 6, e93; doi:10.1038/am.2014.9; published online 21 March 2014

Keywords: cellulose nanofiber paper; coating process; flexible electronics; paper electronics; transparent conductive film

\section{INTRODUCTION}

Transparent conductive films have played essential roles in various electronic applications, including electronic displays and solar cells. ${ }^{1}$ Recently, flexible electronics has become the focus of major research, because it offers new possibilities for next-generation devices with lightweight and portable electronics. ${ }^{2-4}$ In flexible electronics, transparent conductive films have generally been prepared by coating transparent conductive materials on transparent flexible plastic films, such as polyethylene terephthalate (PET). Indium tin oxide is a typical transparent conductive material, and is traditionally used for widespread applications. ${ }^{5}$ However, indium tin oxide is difficult to use in flexible electronics, because it is brittle and breaks easily after bending. ${ }^{6,7}$ Therefore, enormous efforts have been made in the research and development of alternative conductors, such as metal nanowires and nanocarbons. ${ }^{5,8,9}$ Silver nanowires (AgNWs) are among the most promising candidates because of their high optical transparency and electrical conductivity. ${ }^{10-13}$ Carbon nanotubes (CNTs) have also attracted considerable attention because of their excellent chemical stability and mechanical flexibility, ${ }^{14-17}$ although their electrical resistance is relatively high. Wet-coating processes have been widely used for deposition of these conductive materials on plastic films because of their advantages in large-area and lowtemperature production. For example, these transparent conductive films have been prepared by drop coating, ${ }^{17,18}$ bar coating ${ }^{13,19,20}$ and spin coating 21,22 of AgNW or CNT dispersions on plastic films.

One of the most significant challenges in the development of highperformance transparent conductive films is improvement of both the optical transmittance and the electrical conductivity. The transparent conductive performance is strongly related to the dispersion state of the conductive nanomaterials over the substrate surface; uniformly connected conductive networks without significant loss of optical transparency offer better performance. ${ }^{23,24}$ In general, conductive nanomaterials, including the AgNW and the CNT, are dispersed in solvents using dispersing agents or chemical modification, and are then coated on the substrate. However, conventional wet-coating processes, such as drop coating and bar coating, inevitably cause selfaggregation and uneven distribution of the suspended materials after the solvent drying process, as shown by the coffee-ring effect. ${ }^{25,26}$ Although uniform coating is essential for excellent transparent conductive performance, the complex and hard-to-control drying process has made uniformity difficult to achieve. Thus, an alternative coating approach that provides high spatial uniformity for the conductive networks over the substrates is urgently required. In addition, strong adhesion between the conductive material and the substrate surface is also essential for mechanical robustness against friction and bending. However, AgNWs show poor adhesion to plastic 
films, ${ }^{11}$ whereas CNTs show relatively strong adhesion. ${ }^{16}$ Therefore, various techniques, including substrate surface modification, ${ }^{12}$ encapsulation with a thin Teflon layer ${ }^{13}$ and irradiation with highdensity pulsed light, ${ }^{27}$ have been investigated. Despite these efforts, there is still a need for further progress in both coating processes and substrate materials to realize high transparent conductivity performance and strong adhesive properties simultaneously.

In our previous studies, we developed a new type of paper substrate based on wood-derived cellulose nanofibers. ${ }^{28,29}$ The cellulose nanofiber paper, called cellulose nanopaper in this study, demonstrated high optical transparency like that of PET films (specular optical transmittance: 86\%) and a low coefficient of thermal expansion comparable with that of typical glass (58 p.p.m. $\left.\mathrm{K}^{-1}\right),{ }^{29}$ and thus showed great potential as a transparent flexible substrate for future electronic device applications. The paper materials are prepared by a papermaking process, in which an aqueous suspension of cellulose fibers is filtered through a wire mesh to form uniformly distributed and randomly oriented fiber networks. ${ }^{30}$ This filtration technique could also be applied as an effective coating process to prepare uniformly connected networks of fiber-shaped conductive materials, such as metal nanowires and CNTs. Here, we present the uniform coating of AgNWs or CNTs on cellulose nanopaper by a simple filtration process. The 'filtration coating' process produced efficiently connected conductive networks embedded in the nanopaper surface. The sheet resistance of these AgNW networks prepared by filtration coating was $12 \Omega$ sq. $^{-1}$ with specular transmittance of $88 \%$ at $\lambda=550 \mathrm{~nm}$ (without including the nanopaper), which far surpassed the performance of networks prepared by conventional coating processes. The conductive networks also showed strong adhesion to the nanopaper, and maintained their excellent conductive properties even after folding, indicating new possibilities for future foldable electronics.

\section{EXPERIMENTAL PROCEDURES}

\section{Materials}

Cellulose nanofibers with width of approximately $15 \mathrm{~nm}$ and length of more than several $\mu \mathrm{m}$ were extracted from softwood chips (Sitka spruce, Picea sitchensis) as follows. ${ }^{29,31}$ Softwood chips were treated with sodium chlorite and potassium hydroxide. Then, an aqueous suspension of the as-prepared pulp $(0.5$ wt. $\%, 21)$ was subjected to treatment by a high-pressure water-jet system (Star Burst, HJP-25005E, Sugino Machine Co., Ltd, Uozu, Japan) equipped with a ball-collision chamber. The pulp suspension was ejected from a nozzle with a diameter of $0.15 \mathrm{~mm}$ under high pressure of $245 \mathrm{MPa}$ with 50cycle repetitions. AgNWs (average diameter of $70 \mathrm{~nm}$, and average length of $8 \mu \mathrm{m}$ ) were synthesized according to a previously reported method, ${ }^{18}$ followed by dispersion in water or ethanol. An aqueous suspension of single-walled CNTs that had been treated with nitric acid (4-5 nm width, $0.5-1.5 \mu \mathrm{m}$ length and $\mathrm{COOH}$ content of 3-6 wt.\%; Aldrich, Ltd., St Louis, MO, USA) was also prepared according to a previous paper. ${ }^{17}$ The PET films (G2, $50 \mu \mathrm{m}$ thick, Teijin DuPont Films Japan, Ltd., Tokyo, Japan) were treated at $150 \mathrm{~V}$ and $10 \mathrm{~mA}$ for $30 \mathrm{~s}$ with a plasma etching device (AP-T02-L, Sekisui Chemical Co., Ltd., Osaka, Japan) to improve their wettability before use.

Preparation of cellulose nanopaper, AgNW@cellulose nanopaper, CNT@cellulose nanopaper, AgNW@PET film and CNT@PET film Cellulose nanopapers, AgNW@nanopapers and CNT@nanopapers with diameters of approximately $75 \mathrm{~mm}$ were prepared using a simple filtration method (Figure 1a). For AgNW@nanopaper and CNT@nanopaper, an aqueous cellulose nanofiber suspension $(0.35 \mathrm{wt} . \%, 40 \mathrm{ml})$ was first dewatered on a membrane filter (A010A090C, mixed cellulose ester membrane, $0.1 \mu \mathrm{m}$ pore diameter, Advantec Toyo Kaisha, Ltd., Tokyo, Japan) under suction filtration for 20 min. Then, an aqueous suspension of AgNWs or CNTs at a specific concentration $(15 \mathrm{ml})$ was added and filtered in the same manner. The wet sheet obtained was covered with a hydrophobic glass plate, treated by hot pressing at $110^{\circ} \mathrm{C}$ for $20 \mathrm{~min}(1.1 \mathrm{MPa})$ and was then peeled from the membrane. Cellulose nanopaper was also prepared without the AgNWs and CNTs. For AgNW@PET and CNT@PET films, drop coating of the AgNWs or CNTs on the PET films was carried out as follows: an aqueous suspension of AgNWs or CNTs $(1.5 \mathrm{ml})$ at a specific concentration was cast on the PET film $(25 \mathrm{~mm} \times 25 \mathrm{~mm})$, followed by drying at $30^{\circ} \mathrm{C}$. Bar coating and spin coating of ethanol suspensions of AgNWs were also conducted for the cellulose nanopaper and the PET film using a Meyer rod (No. 6) and a spin coater (400-4000 r.p.m., 30 s, 1H-DX2, Mikasa Co., Ltd., Tokyo, Japan), respectively. In all cases, hot pressing treatment was carried out at $110^{\circ} \mathrm{C}$ for $20 \mathrm{~min}$ (at $1.1 \mathrm{MPa}$ ).

\section{Analyses}

The specular optical transmittance was measured with a V-670 spectrophotometer (Jasco, Tokyo, Japan) using a substrate as a reference. The haze was measured using a haze meter (HZ-V3, Suga Test Instruments Co., Ltd., Tokyo, Japan). Sheet resistance values were measured using a resistivity meter with a four-point probe (Loresta-GP, MCP-T610, Mitsubishi Chemical Analytech Co., Ltd., Chigasaki, Japan). The adhesion testing was carried out as follows: an adhesive tape (Nichiban, Tokyo, Japan) was firmly pressed onto the conductive networks on the substrate and then peeled off the samples, followed by measurement of sheet resistance values. This process was repeated ten times, and the adhesion properties of the AgNWs and CNTs on the nanopapers and the PET films were evaluated on the basis of the changes in their sheet resistance values. Surface observations of the nanopapers were conducted using the field emission-scanning electron microscope (FE-SEM, JSM-6700F, JEOL, Ltd., Tokyo, Japan) with an accelerating voltage of $1.5 \mathrm{kV}$. The root mean square values of sample surface roughness were analyzed using a scanning probe microscope (Nanocute, SII NanoTechnology Inc., Tokyo, Japan) in tapping mode (measurement range: $10 \mu \mathrm{m} \times 10 \mu \mathrm{m}$ ).

\section{RESULTS AND DISCUSSION}

The cellulose nanopaper was prepared from mechanically nanofibrillated cellulose. ${ }^{29}$ Traditional white paper made from micro-sized cellulose fibers causes light scattering because of its highly porous microstructure. In contrast, nanopaper prepared from cellulose nanofibers has a densely packed nanostructure, allowing the light scattering to be suppressed. ${ }^{29}$ In this study, nanopapers with thicknesses of approximately $20 \mu \mathrm{m}$ were prepared using cellulose nanofibers with widths of approximately $15 \mathrm{~nm}$, and demonstrated high optical transparency (specular transmittance at $\lambda=550 \mathrm{~nm}$, $T_{550 \mathrm{~nm}}$, of $85 \%$, haze of $6.1 \%$ ). Filtration coating of AgNWs or CNTs onto the nanopaper was conducted via a two-step filtration process (Figure 1a). First, an aqueous dispersion of cellulose nanofibers was filtered through a commercial membrane filter (pore diameter of $0.1 \mu \mathrm{m}$ ) for $20 \mathrm{~min}$ to form a wet nanopaper (nanofiber gel). Second, either the AgNW or the CNT aqueous dispersion was poured on the wet nanopaper, followed by filtration dewatering for $20 \mathrm{~min}$. During the second filtration process, the wet nanopaper acted as an effective filter and retained almost all the conductive nanomaterials on the surface. Finally, the material obtained was thoroughly dried by hot pressing at $110^{\circ} \mathrm{C}$ for $20 \mathrm{~min}(1.1 \mathrm{MPa})$, and was then peeled from the membrane filter. After drying, the wet nanopaper became a transparent flexible substrate. The as-prepared AgNW or CNT networks on the nanopaper, denoted in this paper by AgNW@nanopaper or CNT@nanopaper, respectively, had high optical transparency (Figure 1b). Specular transmittance spectra as a function of wavelength for original nanopaper and AgNW@ nanopaper are shown in Supplementary Figure S1.

Figure 2a shows the transparent conductive performances of AgNW networks on nanopapers and PET films prepared by different coating methods. In each case, hot pressing treatment was conducted after coating under the same conditions. The specular optical 
a

Aqueous dispersion of
AgNW CNT
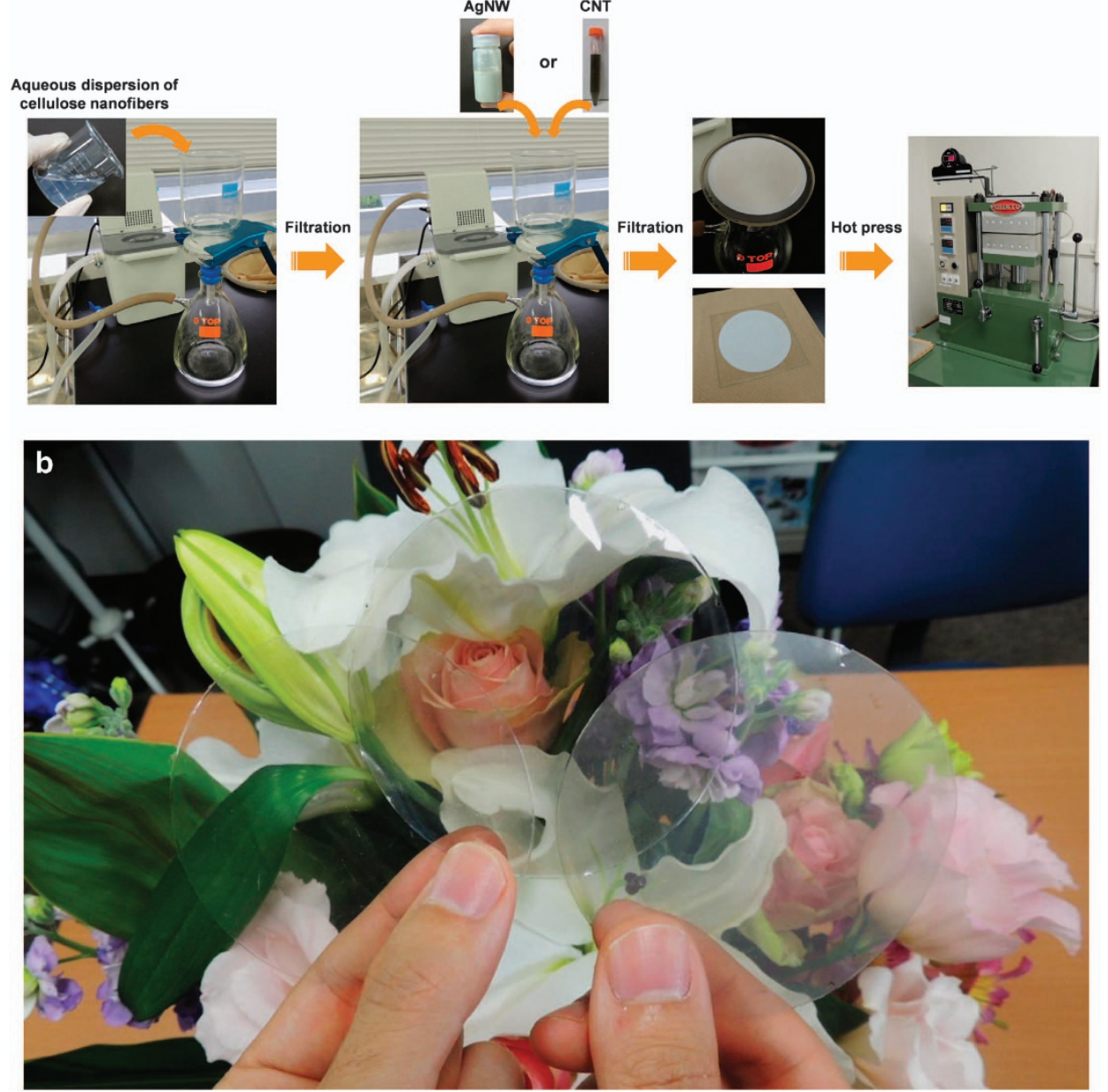

Figure 1 (a) Schematic procedure of filtration coating of AgNW or CNT on the nanopaper. (b) Optical images of original nanopaper (left), CNT@nanopaper (middle) and AgNW@nanopaper (right). Paper size: $75 \mathrm{~mm}$ in diameter. AgNW, silver nanowire; CNT, carbon nanotube.
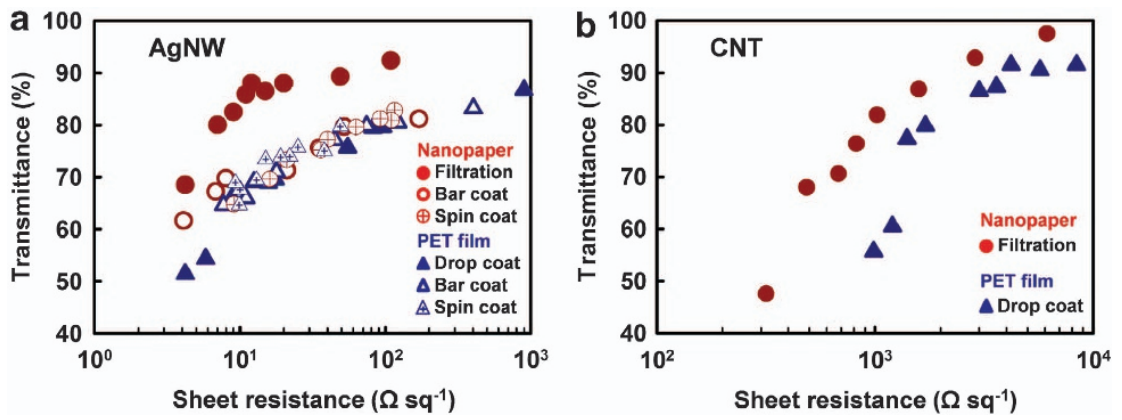

Figure 2 Specular transmittance at $\lambda=550 \mathrm{~nm}$ as a function of sheet resistance for (a) AgNW@nanopapers prepared by filtration coating, bar coating and spin coating, and AgNW@PET films prepared by drop coating, bar coating and spin coating, (b) CNT@nanopaper prepared by filtration coating and CNT@PET film prepared by drop coating. In each case, transmittance values were evaluated with the substrate as a reference. AgNW, silver nanowire; CNT, carbon nanotube; PET, polyethylene terephthalate.

transmittance was evaluated using a substrate as reference. The AgNW networks on the nanopaper prepared by bar coating and spin coating showed sheet resistances of 52 and $63 \Omega \mathrm{sq}^{-1}$, respectively, with $T_{550 \mathrm{~nm}}$ of around $80 \%$. These values were comparable with those of the networks on PET films prepared by bar coating $\left(74 \Omega\right.$ sq. $\left.{ }^{-1}\right)$, spin coating ( $49 \Omega$ sq. $\left.{ }^{-1}\right)$ and drop coating $\left(82 \Omega\right.$ sq. $\left.{ }^{-1}\right)$, indicating that these coating processes make little difference to the transparent conductive properties, regardless of the substrate material. It should be noted that AgNW@nanopaper prepared by filtration coating demonstrated much higher performance; sheet resistance of $12 \Omega$ sq. ${ }^{-1}$ with $T_{550 \mathrm{~nm}}$ of $88 \%$ was achieved, which was 75 times lower than the sheet resistance of PET films prepared by drop coating (900 $\Omega$ sq. ${ }^{-1}$ with $T_{550 \mathrm{~nm}}$ of $87 \%$ ). This transparent conductive performance was superior to those of the state-of-the-art materials 
prepared by various techniques, such as indium tin oxide, graphene, CNT, copper NW, poly(3,4-ethylenedioxythiophene):poly(styrenesulfonate) (Supplementary Figure S2), indicating that AgNW@nanopaper prepared by filtration coating is expected as high-performance transparent conductive films for a broad range of electronic applications. As shown in Figure 2b, these results were confirmed even in the CNT case, where CNT networks on nanopaper prepared by filtration coating showed higher performance than those on PET films prepared by drop coating. Thus, both the AgNW and CNT networks prepared by filtration coating offered much higher transparent conductive performance properties than those prepared by conventional coating processes. Figure 3 displays FE-SEM images of the surfaces of AgNW@nanopaper and AgNW@PET films prepared by different coating methods. In the drop coating case for PET films, the aggregation and non-uniform distribution of the AgNWs were clearly observed (Figure $3 \mathrm{~b}$ ). The bar coating process also led to aggregation, regardless of the substrate material (Figures $3 c$ and $d$ ). Spin coating provided a highly uniform but uniaxially oriented AgNW distribution (Figures $3 \mathrm{e}$ and $\mathrm{f}$ ). Thus, the conventional coating processes would result in partial loss of the connection between the AgNWs. In contrast, filtration coating allowed uniformly distributed and

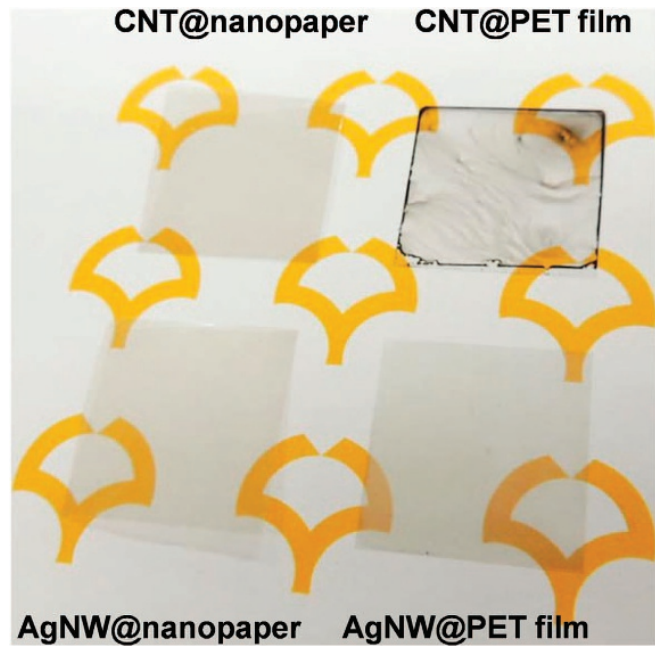

Figure 4 Optical images of CNT@nanopaper and AgNW@nanopaper prepared by filtration coating, CNT@PET film and AgNW@PET film prepared by drop coating. Sample size: $25 \mathrm{~mm} \times 25 \mathrm{~mm}$. AgNW, silver nanowire; CNT, carbon nanotube; PET, polyethylene terephthalate.
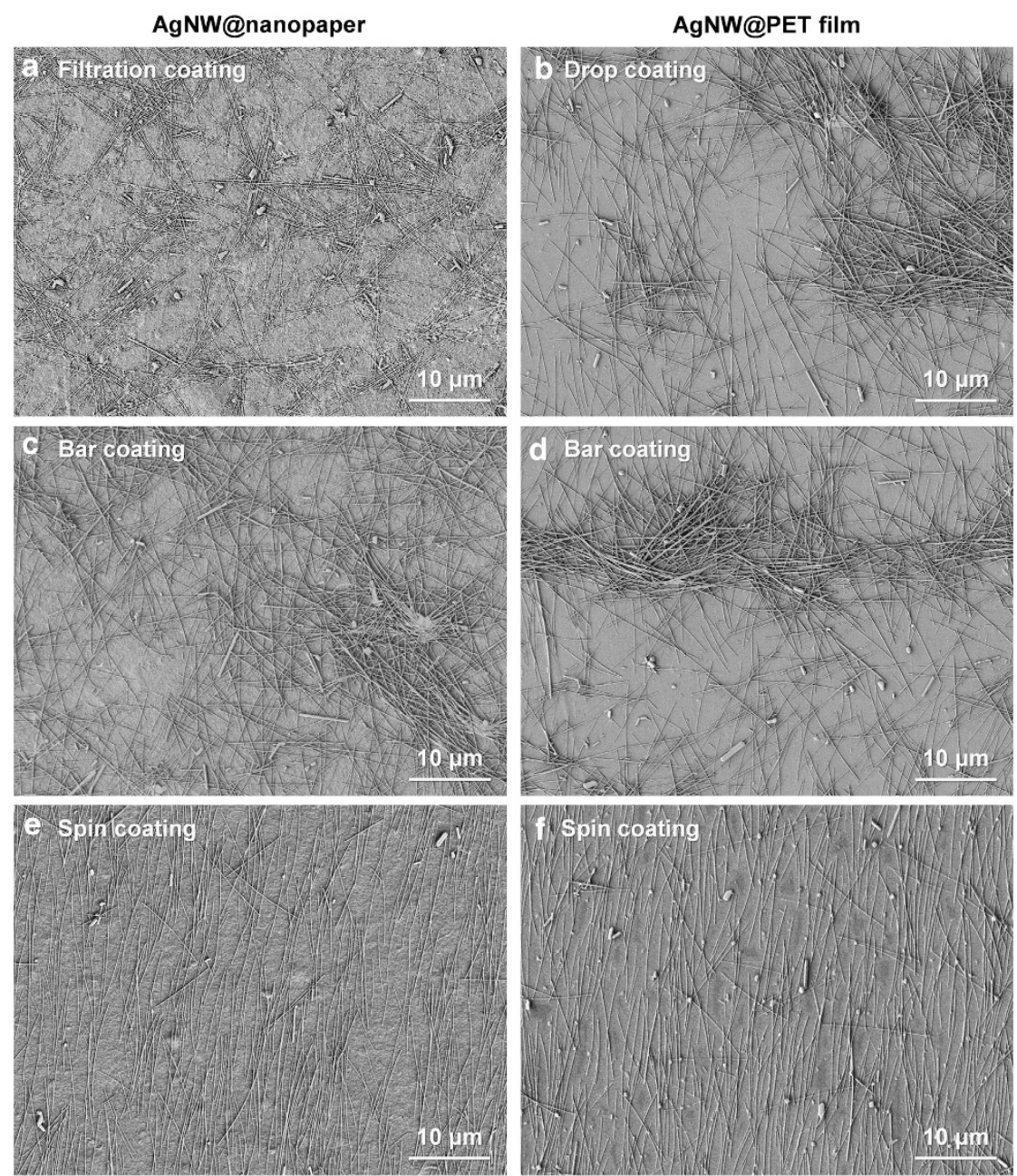

Figure 3 Top-view FE-SEM images of AgNW@nanopaper prepared by (a) filtration coating, (c) bar coating and (e) spin coating, and AgNW@PET film prepared by (b) drop coating, (d) bar coating and (f) spin coating. AgNW, silver nanowire; FE-SEM, field emission-scanning electron microscopy; PET, polyethylene terephthalate. 
randomly oriented AgNW networks, as shown in Figure 3a. Similar tendencies were visually confirmed for CNTs; the coffee-ring phenomenon and an uneven CNT distribution were observed for drop coating on the PET film, whereas the uniform appearance of the CNT distribution was ascertained for filtration coating on nanopaper (Figure 4). These results indicate that the uniformly distributed
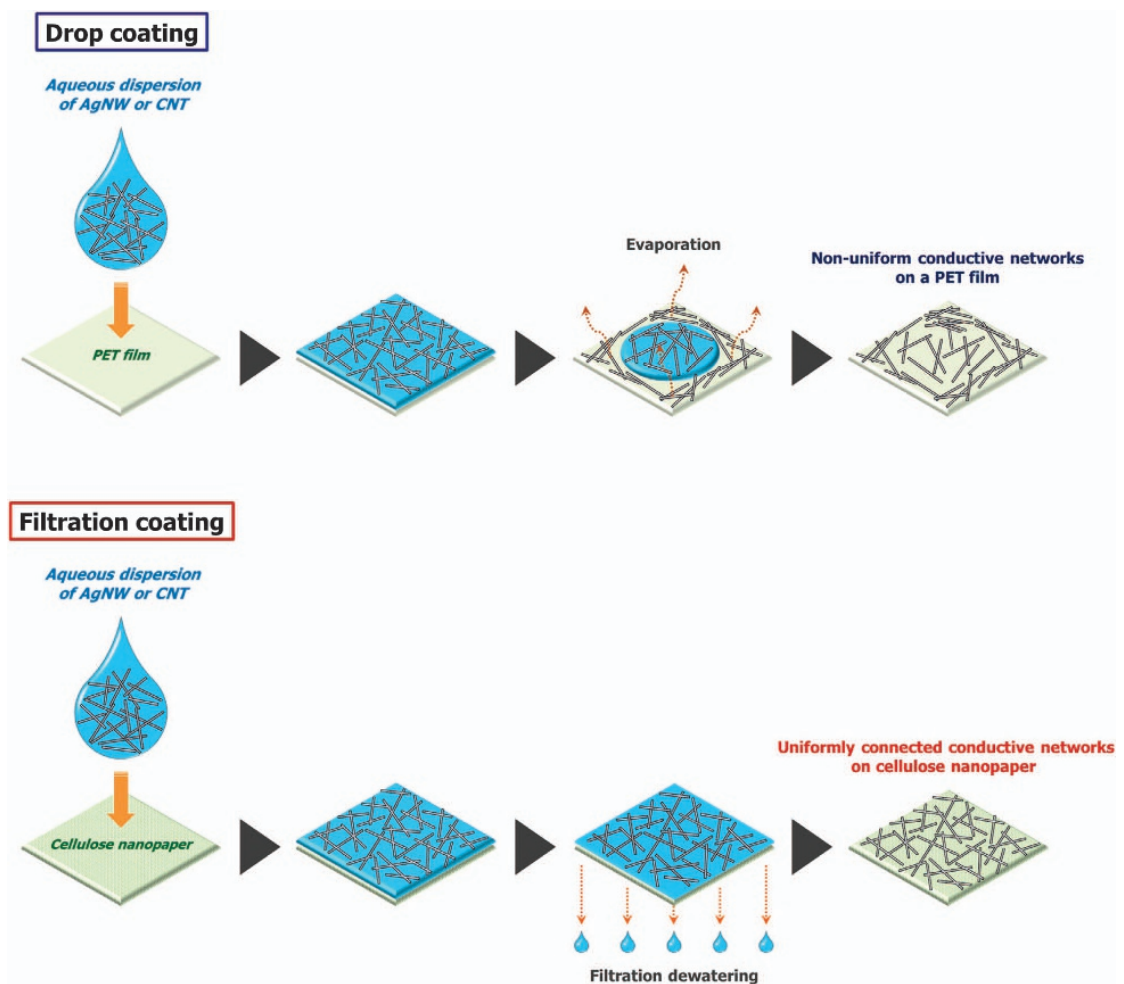

Figure 5 Putative process of the formation of AgNW or CNT networks on PET film and nanopaper by drop coating and filtration coating, respectively. AgNW, silver nanowire; CNT, carbon nanotube; PET, polyethylene terephthalate.
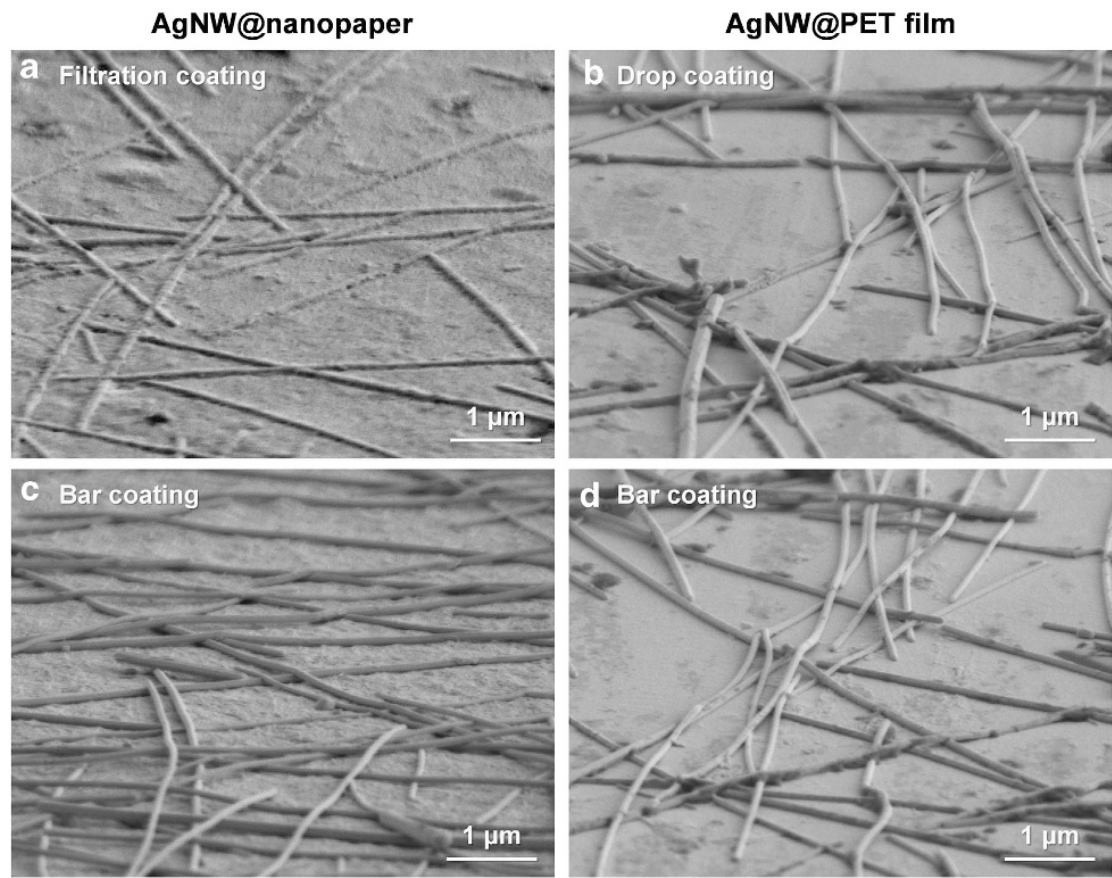

Figure 6 Side-view FE-SEM images of AgNW@nanopaper prepared by (a) filtration coating and (c) bar coating, and AgNW@PET film prepared by (b) drop coating and (d) bar coating. AgNW, silver nanowire; FE-SEM, field emission-scanning electron microscopy; PET, polyethylene terephthalate. 

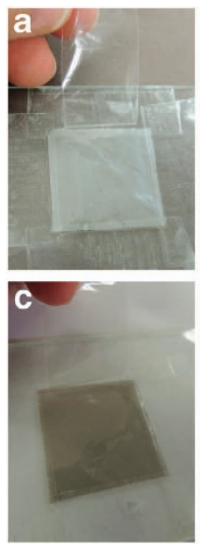
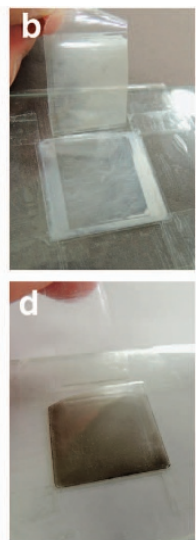

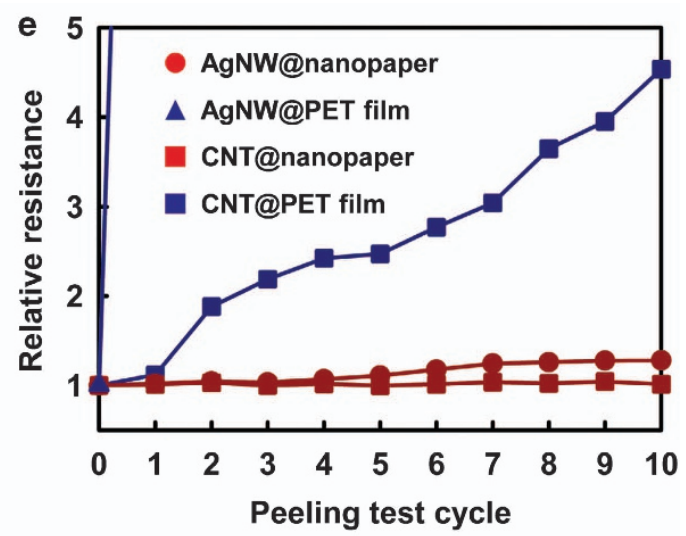

Figure 7 Optical images of (a) AgNW@nanopaper, (b) AgNW@PET film, (c) CNT@nanopaper and (d) CNT@PET film after the peeling test. AgNW@nanopaper and CNT@nanopaper were prepared by filtration coating. AgNW@PET film and CNT@PET film were prepared by drop coating. (e) Relative resistance values of AgNW@nanopaper and CNT@nanopaper prepared by filtration coating, and AgNW@PET film and CNT@PET film prepared by drop coating as a function of peeling test cycles. AgNW, silver nanowire; CNT, carbon nanotube; FE-SEM, field emission-scanning electron microscopy; PET, polyethylene terephthalate.
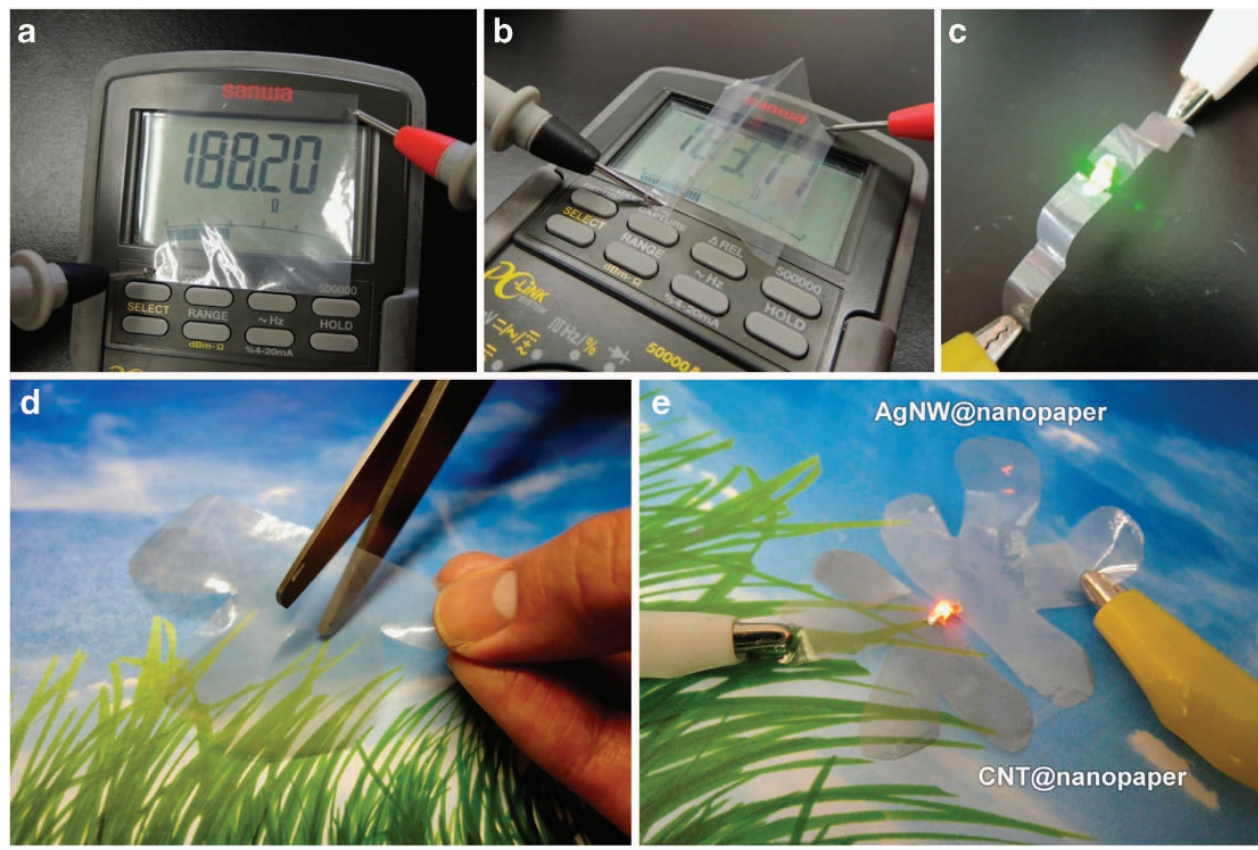

Figure 8 Flexible performance of the transparent conductive papers. Resistance values of AgNW@nanopaper (a) before (188 $\Omega$ ) and (b) after (184 $\Omega$ ) mountain folding. (c) The lighting of a green LED placed between mountain- and valley-folded AgNW@nanopapers. (d and e) Paper craft by using transparent conductive papers. AgNW, silver nanowire; LED, light-emitting diode.

random conductive networks formed by filtration coating are efficiently interconnected, providing excellent transparent conductive performance. The differences in the AgNW and CNT distribution states on the substrates between the different coating techniques are related to the suspension drying processes. The proposed mechanism is discussed as follows (Figure 5). In the case of the drop coating process for PET films, an aqueous dispersion of AgNWs or CNTs was cast on the PET and then gently dried. During the drying process, the water evaporated in the direction from the edge to the center of the PET film. The suspended conductive nanomaterials thus moved to the drying front of the wet film, leading to their self-aggregation after complete drying (Figure $3 \mathrm{~b}$ ), as in the well-known coffee-ring effect. $^{25,26}$ In the bar coating process case using an ethanol dispersion of $\mathrm{AgNW}, \mathrm{AgNW}$ agglomeration was inevitable because of the rapid and uneven evaporation of the ethanol solvent (Figures $3 c$ and d). The spin coating process resulted in a uniform but unidirectional AgNW distribution because of the centrifugal force (Figures $3 e$ and $\mathrm{f}$ ). Overall, it is difficult to control the network quality with the conventional coating processes, leading to relatively low transparent conductive performance. In contrast, filtration coating of the nanopaper allowed homogeneous deposition of the conductive nanomaterials, as a dispersion state in water, over the nanopaper surface (Figure 3a) because of the perpendicular draining process by filtration through the paper-specific nanopores. Thus, this 
simple filtration process, which was made feasible by using the nanopaper substrate, was an effective coating process, providing efficiently connected conductive networks to achieve high transparent conductive performance.

Figure 6 shows side-view FE-SEM images of AgNW networks on the surfaces of the nanopaper and a PET film prepared by different coating methods. It can be seen that the AgNW networks were embedded in the surface layer of the nanopaper only in the filtration coating case. Although the physical strength of nanopaper is high in the dry state (Young's modulus of $13 \mathrm{GPa}$; tensile strength of $223 \mathrm{MPa}){ }^{28}$ it decreases dramatically in the wet state, because water molecules penetrate the paper and break the hydrogen bonds between the cellulose nanofibers. Therefore, this embedding phenomenon possibly occurred because the hot pressing treatment of the AgNW networks was conducted on wet nanopaper in the filtration coating process, whereas it was carried out on dry nanopaper or PET films in the other coating processes. The root mean square values of surface roughness of the AgNW@nanopaper prepared by filtration coating was approximately $30 \mathrm{~nm}$, which was 2.5 times lower than that obtained by bar coating (around $75 \mathrm{~nm}$ ). Then, the haze value of the AgNW@nanopaper prepared by filtration coating (12.16\%) was lower than that prepared by bar coating (14.50\%). Hence, this smooth surface would contribute to suppression of light scattering and improved specular transmittance, leading to high transparent conductive performance. The embedded AgNW networks also showed strong adhesion to the nanopaper surface. Figures $7 \mathrm{a}$ and $\mathrm{b}$ shows optical images of the AgNW@nanopaper and AgNW@PET films, respectively, after peeling tests using adhesive tape. The AgNW networks on the PET film were easily peeled off with the tape (Figure 7b), resulting in significantly increased sheet resistance (Figure 7e). In contrast, the AgNW networks prepared by filtration coating adhered strongly to the nanopaper surface (Figure 7a); the sheet resistance remained almost unchanged, even after ten peeling test cycles (Figure 7e). The CNT networks also adhered more strongly to the nanopaper than to the PET film (Figures $7 \mathrm{c}-\mathrm{e}$ ), indicating that a $\mathrm{CH}-\pi$ interaction between the axial plane of the cellulose and the graphene $\pi$-conjugated system ${ }^{32}$ improves the adhesion of CNT networks to the nanopaper surface. Thus, highly transparent and strongly adhesive conductive networks were successfully formed on the transparent nanopaper surface by the simple filtration coating process.

As shown in Figures $8 \mathrm{a}-\mathrm{c}$, these transparent conductive papers were foldable beyond the point of mere flexibility. The resistance value of the AgNW@nanopaper remained almost the same even after mountain-shaped folding, and the multiply folded papers were used to light a light-emitting diode. The papers could easily be cut with scissors for versatile shape design, allowing transparent conductive paper craft (Figures $8 \mathrm{~d}$ and e). The excellent flexibility performance of the transparent conductive papers may open new avenues for future foldable electronics.

\section{CONCLUSIONS}

In conclusion, we have demonstrated the fabrication of highly transparent conductive networks on cellulose nanopaper using a simple filtration process. The cellulose nanopaper, which was prepared from the most ubiquitous and abundant biological resources, acted as both filter and transparent flexible substrate for the AgNWs and CNTs. The filtration coating process described here can be applied to various conductive materials and can also be extended to large-area production like a well-established papermaking process. This novel concept breaks new ground in the creation of next-generation paper electronics.

\section{ACKNOWLEDGEMENTS}

HK and MN were supported by the Japan Science and Technology Agency A-Step Feasibility Study Program (JST A-Step, Japan) and the Funding Program for Next Generation World-Leading Researchers (NEXT Program, Japan), respectively.

1 Ginley, D. S., Hosono, H. \& Paine, D. C. Handbook of Transparent Conductors (eds Ginley, D. S., Hosono, H. \& Paine, D. C.) (Springer, New York, NY, USA, 2010).

2 Nathan, A., Ahnood, A., Cole, M. T., Sungsik, L., Suzuki, Y., Hiralal, P., Bonaccorso, F., Hasan, T., Garcia-Gancedo, L., Dyadyusha, A., Haque, S., Andrew, P., Hofmann, S., Moultrie, J., Chu, D., Flewitt, A. J., Ferrari, A. C., Kelly, M. J., Robertson, J., Amaratunga, G. A. J. \& Milne, W. I. Flexible electronics: the next ubiquitous platform. Proc. IEEE 100, 1486-1517 (2012).

3 Wong, W. S. \& Salleo, A. Flexible Electronics: Materials and Applications (eds Wong, W. S. \& Salleo, A.) (Springer, New York, NY, USA, 2009).

4 Tobjörk, D. \& Österbacka, R. Paper electronics. Adv. Mater. 23, 1935-1961 (2011).

5 Ellmer, K. Past achievements and future challenges in the development of optically transparent electrodes. Nat. Photon. 6, 809-817 (2012).

6 Chen, Z., Cotterell, B., Wang, W., Guenther, E. \& Chua, S. A mechanical assessment of flexible optoelectronic devices. Thin Solid Films 394, 201-205 (2001).

7 Chen, Z., Cotterell, B. \& Wang, W. The fracture of brittle thin films on compliant substrates in flexible displays. Eng. Fract. Mech. 69, 597-603 (2002).

8 Kumar, A. \& Zhou, C. The race to replace tin-doped indium oxide: which material will win? ACS Nano 4, 11-14 (2010).

9 Hecht, D. S., Hu, L. \& Irvin, G. Emerging transparent electrodes based on thin films of carbon nanotubes, graphene, and metallic nanostructures. Adv. Mater. 23, 1482-1513 (2011).

10 Lee, J.-Y., Connor, S. T., Cui, Y. \& Peumans, P. Solution-processed metal nanowire mesh transparent electrodes. Nano Lett. 8, 689-692 (2008).

11 De, S., Higgins, T. M., Lyons, P. E., Doherty, E. M., Nirmalraj, P. N., Blau, W. J., Boland, J. J. \& Coleman, J. N. Silver nanowire networks as flexible, transparent, conducting films: extremely high DC to optical conductivity ratios. ACS Nano 3, 1767-1774 (2009).

12 Madaria, A. R., Kumar, A., Ishikawa, F. N. \& Zhou, C. Uniform, highly conductive, and patterned transparent films of a percolating silver nanowire network on rigid and flexible substrates using a dry transfer technique. Nano Res. 3, 564-573 (2010).

13 Hu, L., Kim, H. S., Lee, J.-Y., Peumans, P. \& Cui, Y. Scalable coating and properties of transparent, flexible, silver nanowire electrodes. ACS Nano 4, 2955-2963 (2010).

14 Zhang, D., Ryu, K., Liu, X., Polikarpov, E., Ly, J., Tompson, M. E. \& Zhou, C. Transparent, conductive, and flexible carbon nanotube films and their application in organic light-emitting diodes. Nano Lett. 6, 1880-1886 (2006).

15 Mirri, F., Ma, A. W. K., Hsu, T. T., Behabtu, N., Eichmann, S. L., Young, C. C. Tsentalovich, D. E. \& Pasquali, M. High-performance carbon nanotube transparent conductive films by scalable dip coating. ACS Nano 6, 9737-9744 (2012).

16 Kim, Y., Chikamatsu, M., Azumi, R., Saito, T. \& Minami, N. Industrially feasible approach to transparent, flexible, and conductive carbon nanotube films: celluloseassisted film deposition followed by solution and photonic processing. Appl. Phys. Express 6, 025101 (2013).

17 Koga, H., Saito, T., Kitaoka, T., Nogi, M., Suganuma, K. \& Isogai, A. Transparent, conductive, and printable composites consisting of TEMPO-oxidized nanocellulose and carbon nanotube. Biomacromolecules 14, 1160-1165 (2013).

18 Tokuno, T., Nogi, M., Karakawa, M., Jiu, J., Nge, T. T., Aso, Y. \& Suganuma, K. Fabrication of silver nanowire transparent electrodes at room temperature. Nano Res. 4, 1215-1222 (2011).

$19 \mathrm{Liu}, \mathrm{C} . \& \mathrm{Yu}, \mathrm{X}$. Silver nanowire-based transparent, flexible, and conductive thin film. Nanoscale Res. Lett. 6, 75 (2011).

20 Dan, B., Irvin, G. C. \& Pasquali, M. Continuous and scalable fabrication of transparent conducting carbon nanotube films. ACS Nano 3, 835-843 (2009).

21 Yim, J. H., Kim, Y. S., Koh, K. H. \& Lee, S. Fabrication of transparent single wall carbon nanotube films with low sheet resistance. J. Vac. Sci. Technol. B 26, 851-855 (2008).

22 Lee, D., Lee, H., Ahn, Y., Jeong, Y., Lee, D.-Y. \& Lee, Y. Highly stable and flexible silver nanowire-graphene hybrid transparent conducting electrodes for emerging optoelectronic devices. Nanoscale 5, 7750-7755 (2013).

23 Groep, J., Spinelli, P. \& Polman, A. Transparent conducting silver nanowire networks. Nano Lett. 12, 3138-3144 (2012).

24 Kim, T. Y., Kim, Y. W., Lee, H. S., Kim, H., Yang, W. S. \& Suh, K. S. Uniformly interconnected silver-nanowire networks for transparent film heaters. Adv. Funct. Mater. 23, 1250-1255 (2013).

25 Deegan, R. D., Bakajin, O., Dupont, T. F., Huber, G., Nagel, S. R. \& Witten, T. A. Capillary flow as the cause of ring stains from dried liquid drops. Nature $\mathbf{3 8 9}$, 827-829 (1997).

26 Han, W. \& Lin, Z. Learning from "coffee rings": ordered structures enabled by controlled evaporative self-assembly. Angew. Chem. Int. Ed. 51, 1534-1546 (2012).

27 Jiu, J., Nogi, M., Sugahara, T., Tokuno, T., Araki, T., Komoda, N., Suganuma, K., Uchida, H. \& Shinozaki, K. Strongly adhesive and flexible transparent silver nanowire conductive films fabricated with a high-intensity pulsed light technique. J. Mater. Chem. 22, 23561-23567 (2012). 
28 Nogi, M., Iwamoto, S., Nakagaito, A. N. \& Yano, H. Optically transparent nanofiber paper. Adv. Mater. 21, 1595-1598 (2009).

29 Nogi, M., Kim, C., Sugahara, T., Inui, T., Takahashi, T. \& Suganuma, K. High thermal stability of optical transparency in cellulose nanofiber paper. Appl. Phys. Lett. 102, 181911 (2013).

30 Sampson, W. W. The structural characterisation of fibre networks in papermaking processes: a review. in Proc. The Science of Papermaking (ed. Baker, C. F.) Trans. 11th Fund. Res. Symp. 1205-1288 (FRC, Manchester, 2001).

31 Abe, K., Iwamoto, S. \& Yano, H. Obtaining cellulose nanofibers with a uniform width of $15 \mathrm{~nm}$ from wood. Biomacromolecules 8, 3276-3278 (2007).
32 Yokota, S., Ueno, T., Kitaoka, T. \& Wariishi, H. Molecular imaging of single cellulose chains aligned on a highly oriented pyrolytic graphite surface. Carbohydr. Res. $\mathbf{3 4 2}$ 2593-2598 (2007).

(c) (i) (2) (2) This work is licensed under a Creative Commons Attribution-NonCommercial-ShareAlike 3.0 Unported License. To view a copy of this license, visit http://creativecommons. org/licenses/by-nc-sa/3.0/

Supplementary Information accompanies the paper on the NPG Asia Materials website (http://www.nature.com/am) 